\title{
Existence of solutions for a coupled system of fractional differential equations by means of topological degree theory
}

Jingli Xie ${ }^{1^{*}}$ (D) and Lijing Duan'

"Correspondence:

xiejingli721124@163.com

${ }^{1}$ College of Mathematics and

Statistics, Jishou University, Jishou,

Hunan 416000, P.R. China

\section{Springer}

\begin{abstract}
This paper investigates the existence of solutions for a coupled system of fractional differential equations. The existence is proved by using the topological degree theory, and an example is given to show the applicability of our main result.

MSC: 34B15; 34B18; 34B37; 58E30

Keywords: Fractional differential equations; Boundary value problems; Topological degree theory; Coupled systems
\end{abstract}

\section{Introduction}

In this manuscript, the following coupled system of fractional differential equations is discussed:

$$
\begin{cases}D^{\theta}[x(t)-f(t, x(t))]=h\left(t, y(t), I^{\alpha} y(t)\right), & t \in[0,1], \\ D^{\theta}[y(t)-f(t, y(t))]=h\left(t, x(t), I^{\alpha} x(t)\right), & t \in[0,1], \\ x^{\prime}(0)=y^{\prime}(0)=0, & \\ a_{1} x(0)-b_{1} x(\eta)-c_{1} x(1)=\frac{1}{\Gamma(\theta)} \int_{0}^{1}(1-s)^{\theta-1} \phi(s, x(s)) d s, \\ a_{2} y(0)-b_{2} y(\xi)-c_{2} y(1)=\frac{1}{\Gamma(\theta)} \int_{0}^{1}(1-s)^{\theta-1} \psi(s, y(s)) d s,\end{cases}
$$

where $1<\theta \leq 2, \alpha>0, \eta, \xi \in(0,1], a_{j}, b_{j}, c_{j}(j=1,2)$ are real numbers with $a_{j} \neq b_{j}+c_{j}$ $(j=1,2)$. Further $f, \phi, \psi:[0,1] \times \mathbb{R} \rightarrow[0,1]$, and $h:[0,1] \times \mathbb{R} \times \mathbb{R} \rightarrow[0,1]$ are continuous functions, $f(0, x(0))=0,\left.\frac{\partial^{i} f(t, x(t))}{\partial t^{i}}\right|_{t=0}=0$ for $i=1,2, \ldots, n-1 . D^{\theta}$ represents the Caputo fractional derivative of order $\theta$.

Fractional differential equations are widely used in many fields such as chemistry, physics, biology, and optimization theory [1-4]. In addition, coupled systems of fractional differential equations have attracted particular concern from scholars considering their appearance in the mathematical modeling of physical phenomena like chaos synchronization [5], anomalous diffusion [6], disease models [7], and so on. The existence theory to fractional differential equations with integral boundary conditions has widespread applications in optimization theory, many researchers have studied [8-13], and the existence

(c) The Author(s) 2021. This article is licensed under a Creative Commons Attribution 4.0 International License, which permits use, sharing, adaptation, distribution and reproduction in any medium or format, as long as you give appropriate credit to the original author(s) and the source, provide a link to the Creative Commons licence, and indicate if changes were made. The images or other third party material in this article are included in the article's Creative Commons licence, unless indicated otherwise in a credit line to the material. If material is not included in the article's Creative Commons licence and your intended use is not permitted by statutory regulation or exceeds the permitted use, you will need to obtain permission directly from the copyright holder. To view a copy of this licence, visit http://creativecommons.org/licenses/by/4.0/. 
of solutions is the basis of studying the stability and numerical solutions of differential equations [14]. For the existence of solutions of fractional differential equations, the authors use diverse methods, such as fixed point theory [15-19], upper and lower solutions method [20], monotone iterative technique and Mawhin's continuation theorem [21], and topological degree theory [22]. When studying the existing literature, we find that fractional differential equations with integral boundary conditions are not properly tested via topological degree theory. Thus we investigate the existence result to a coupled system of fractional differential equations(1.1) through applying topological degree theory.

Bashiri et al. [23]investigated the existence of solutions for fractional differential equations by means of the coupled fixed point theorem of Krasnoselskii type

$$
\left\{\begin{array}{l}
D^{\theta}[x(t)-f(t, x(t))]=h\left(t, y(t), I^{\alpha} y(t)\right), \\
D^{\theta}[y(t)-f(t, y(t))]=h\left(t, x(t), I^{\alpha} x(t)\right), \\
x(0)=y(0)=0,
\end{array}\right.
$$

where $D^{\theta}$ denotes the Riemann-Liouville fractional derivative, $\theta \in(0,1), \alpha>0$.

Ahmad et al. [24] established existence results as well as studied qualitative aspects of the proposed coupled system of fractional hybrid delay differential equations

$$
\begin{cases}{ }^{C} D_{+0}^{\kappa}\left(r(t)-P_{1}(t, r(t), h(t))\right)=Q_{1}(t, r(v t), h(v t)), & t \in \mathcal{A}, \\ { }^{C} D_{+0}^{\sigma}\left(h(t)-P_{2}(t, r(t), h(t))\right)=Q_{2}(t, r(v t), h(v t)), & t \in \mathcal{A}, \\ \left.r(t)\right|_{t=0}=r_{0},\left.\quad h(t)\right|_{t=0}=h_{0}, & \end{cases}
$$

where $\mathcal{A}=[0, \tau],{ }^{C} D_{+0}, \tau>0$ is Caputo's derivative, and $r_{0}, h_{0}$ are real numbers, while the delay parameter is denoted by $v \in(0,1)$.

Muthaiah et al. [25]considered the existence and Hyers-Ulam type stability results for the nonlinear coupled system of Caputo-Hadamard type fractional differential equations

$$
\left\{\begin{array}{l}
{ }^{C} D^{\varrho} y(\tau)=f(\tau, y(\tau), z(\tau)), \quad \tau \in[1, T]:=\mathcal{K}, \\
{ }^{C} D^{\varsigma} z(\tau)=g(\tau, y(\tau), z(\tau)), \quad \tau \in[1, T]:=\mathcal{K}, \\
y(1)=0, y^{\prime}(1)=0, y(T)=\alpha_{1} \sum_{j=1}^{k-2} \xi_{j} z\left(\zeta_{j}\right)+\beta_{1}{ }^{H} I^{\varsigma 1} z(\vartheta), \\
z(1)=0, z^{\prime}(1)=0, z(T)=\alpha_{2} \sum_{j=1}^{k-2} v_{j} z\left(\omega_{j}\right)+\beta_{2}{ }^{H} I^{\varrho} y(\varphi), \\
1<\vartheta<\varphi<\xi_{1}<\omega_{1}<\xi_{2}<\omega_{2}<\cdots<\xi_{k-2}<\omega_{k-2}<T,
\end{array}\right.
$$

where ${ }^{C} D^{(\cdot)}$ denotes the Caputo-Hadamard fractional derivative, ${ }^{H} I^{(\cdot)}$ denotes the Hadamard fractional integrals, $2<\varrho, \varsigma \leq 3,0<\varrho_{1}, \varsigma_{1}<1, \alpha_{1}, \alpha_{2}, \beta_{1}, \beta_{2}$ are real constants and $\zeta_{j}, v_{j}, j=1,2, \ldots, k-2$, are positive real constants. The consequence of existence is obtained by employing the alternative of Leray-Schauder and Krasnoselskii's, whereas the uniqueness result is based on the principle of Banach contraction mapping.

Motivated especially by the aforementioned work, we consider the existence of solutions to a coupled system of fractional differential equations (1.1). According to our literature review, no scholars have studied equation (1.1), the results are entirely new. The remainder of this paper is as follows. In the second part, we display some definitions, facts, and results. We confirm the existence of solutions for system (1.1) in the third part. Finally, we provide an example to prove our results. 


\section{Preliminaries}

In this part, we recollect a number of facts, definitions, and conclusions. Let $C([0,1] \times$ $\mathbb{R},[0,1])$ represent the space of all continuous functions $f, \phi, \psi:[0,1] \times \mathbb{R} \rightarrow[0,1]$, and let $C([0,1] \times \mathbb{R} \times \mathbb{R},[0,1])$ express the class of functions $h:[0,1] \times \mathbb{R} \times \mathbb{R} \rightarrow[0,1]$ such that

(1) the map $t \rightarrow h(t, x, y)$ is measurable for each $x, y \in \mathbb{R}$,

(2) the map $x \rightarrow h(t, x, y)$ is continuous for each $x \in \mathbb{R}]$,

(3) the map $y \rightarrow h(t, x, y)$ is continuous for each $y \in \mathbb{R}$.

Let $X$ be a Banach space and $\mathbb{B} \subset P(X)$, where $P(X)$ stands for the family of all bounded subsets of $X$. Next, we introduce some concepts.

Definition 2.1 ([26]) The Kuratowski measure of noncompactness $\alpha: \mathbb{B} \rightarrow \mathbb{R}_{+}$is defined as

$\alpha(B)=\inf \{d>0$, where $B \in \mathbb{B}$ admits a finite cover by set of diameter $\leq d\}$.

Definition 2.2 ([26]) Let $\mathcal{F}: \Omega \rightarrow X$ be a continuous bounded map, where $\Omega \subseteq X$. Then $\mathcal{F}$ is

(1) $\alpha$-Lipschitz if there exists $k \geq 0$, therefore $\alpha(\mathcal{F}(S)) \leq k \alpha(S)$ for all bounded subsets $S \subseteq \Omega$

(2) strict $\alpha$-contraction if there exists $0 \leq k<1$ such that $\alpha(\mathcal{F}(S)) \leq k \alpha(S)$ for all bounded subsets $S \subseteq \Omega$;

(3) $\alpha$-condensing if $\alpha(\mathcal{F}(S))<\alpha(S)$ for all bounded subsets $S \subseteq \Omega$ with $\alpha(S)>0$. In other words, $\alpha(\mathcal{F}(S)) \geq \alpha(S)$ implies $\alpha(S)=0$.

All classes of strict $\alpha$-contraction $\mathcal{F}: \Omega \rightarrow X$ and all classes of $\alpha$-condensing maps $\mathcal{F}$ : $\Omega \rightarrow X$ are represented by $\Lambda C_{\alpha}(\Omega)$ and $C_{\alpha}(\Omega)$, respectively. Then $\Lambda C_{\alpha}(\Omega) \subset C_{\alpha}(\Omega)$ and each $\mathcal{F} \in C_{\alpha}(\Omega)$ is $\alpha$-Lipschitz with constant $k=1$. Moreover, $\mathcal{F}: \Omega \rightarrow X$ is Lipschitz whenever there is $k>0$, therefore

$$
\|\mathcal{F}(x)-\mathcal{F}(y)\| \leq k\|x-y\| \quad \text { for all } x, y \in \Omega
$$

Further, $\mathcal{F}$ will be a strict contraction if $k<1$.

Proposition 2.3 ([27]) If $\mathcal{F}, \mathcal{G}: \Omega \rightarrow X$ are $\alpha$-Lipschitz with respective constants $k_{1}$ and $k_{2}$, then $\mathcal{F}+\mathcal{G}$ is $\alpha$-Lipschitz with constant $k_{1}+k_{2}$.

Proposition 2.4 ([27]) If $\mathcal{F}: \Omega \rightarrow X$ is Lipschitz with constant $k$, then $\mathcal{F}$ is $\alpha$-Lipschitz with the equal constant $k$.

Proposition 2.5 ([27]) If $\mathcal{F}: \Omega \rightarrow X$ is compact, then $\mathcal{F}$ is $\alpha$-Lipschitz with constant $k=0$.

Theorem 2.6 ([27]) If $\mathcal{F}: X \rightarrow X$ is $\alpha$-condensing and

$$
\Lambda=\{x \in X \text { : there exists } 0 \leq v \leq 1 \text { such that } x=v \mathcal{F} x\} .
$$

If $\Lambda$ is a bounded set in $X$, so we have $r>0$ such that $\Lambda \subset B_{r}(0)$, then

$$
\left.D\left(I-\nu \mathcal{F}, B_{r}(0), 0\right)\right)=1 \quad \text { for all } v \in[0,1] .
$$


Consequently, $\mathcal{F}$ has at least one fixed point, and the set of the fixed points of $\mathcal{F}$ lies in $B_{r}(0)$.

Definition 2.7 ([28]) The fractional integral of order $\theta(\theta>0)$ of function $f:[0, \infty) \rightarrow R$ is defined as

$$
I^{\theta} f(t)=\frac{1}{\Gamma(\theta)} \int_{0}^{t}(t-s)^{\theta-1} f(s) d s,
$$

provided that the right-hand side is pointwise defined on $(0, \infty)$.

Definition 2.8 ([28]) The Caputo fractional derivative of order $\theta(\theta>0)$ of the function $f:[0, \infty) \rightarrow R$ is defined by

$$
D^{\theta} f(t)=\frac{1}{\Gamma(n-\theta)} \int_{0}^{t}(t-s)^{n-\theta-1} f^{(n)}(s) d s
$$

where $t>0, n=[\theta]+1$.

Lemma 2.9 ([28]) Let $\theta>0$, then the following result holds for fractional differential equations:

$$
I^{\theta}\left[D^{\theta} f(t)\right]=f(t)+C_{0}+C_{1} t+C_{2} t^{2}+\cdots+C_{n-1} t^{n-1}
$$

for arbitrary $n=[\theta]+1,[\theta]$ indicates the integer part of the real number $\theta>0, C_{i} \in \mathbb{R}$, $i=0,1,2, \ldots, n-1 . D^{\theta}$ is a Caputo fractional derivative.

\section{Main results}

In this part, we discuss the existence result for (1.1).

The space $X=C([0,1], \mathbb{R})$ of all continuous functions is a Banach space under the topological norm $\|x\|=\sup \{|x(t)|: t \in[0,1]\}$ and the product space $X \times X$ is a Banach space under the norm $\|(x, y)\|=\|x\|+\|y\|$ or $\|(x, y)\|=\max \{\|x\|,\|y\|\}$.

In order to get the result of our result, we need the following hypotheses.

$\left(H_{1}\right)$ For each $(t, x),(t, \bar{x}),(t, y),(t, \bar{y}) \in[0,1] \times \mathbb{R}$, there exist constants $\lambda_{1}, \lambda_{2} \in[0,1)$ such that

$$
\begin{gathered}
|f(t, x)-f(t, \bar{x})| \leq \lambda_{1}\|x-\bar{x}\|, \\
|f(t, y)-f(t, \bar{y})| \leq \lambda_{2}\|y-\bar{y}\| .
\end{gathered}
$$

$\left(H_{2}\right)$ For each $(t, x, y) \in \mathbb{R}$, there exist positive constants $l_{h}^{1}, l_{h}^{2}, M_{h}$ and $q_{1} \in[0,1)$ such that

$$
|h(t, x, y)| \leq l_{h}^{1}\|x\|^{q_{1}}+l_{h}^{2}\|y\|^{q_{1}}+M_{h} .
$$

$\left(H_{3}\right)$ For each $(t, x) \in[0,1] \times \mathbb{R}$, there exist positive constants $l_{f}, M_{f}$ and $q_{2} \in[0,1)$ such that

$$
|f(t, x(t))| \leq l_{f}\|x\|^{q_{2}}+M_{f}
$$


$\left(H_{4}\right)$ For each $(t, x),(t, y) \in[0,1] \times \mathbb{R}$, there exist positive constants $c_{\phi}, c_{\psi}, M_{\phi}, M_{\psi}$, and $q_{2} \in[0,1)$ such that

$$
\begin{gathered}
|\phi(t, x)| \leq c_{\phi}\|x\|^{q_{2}}+M_{\phi}, \\
|\psi(t, y)| \leq c_{\psi}\|y\|^{q_{2}}+M_{\psi} .
\end{gathered}
$$

$\left(H_{5}\right)$ For each $(t, x),(t, \bar{x}),(t, y),(t, \bar{y}) \in[0,1] \times \mathbb{R}$, we have positive constants $b_{\phi}, b_{\psi} \in[0,1)$ such that

$$
\begin{gathered}
|\phi(t, x)-\phi(t, \bar{x})| \leq b_{\phi}\|x-\bar{x}\|, \\
|\psi(t, y)-\psi(t, \bar{y})| \leq b_{\psi}\|y-\bar{y}\| .
\end{gathered}
$$

Lemma 3.1 If $f(0, x(0))=0,\left.\frac{\partial^{i} f(t, x(t))}{\partial t^{i}}\right|_{t=0}=0$ for $i=1,2, \ldots, n-1$, then the consequence of fractional differential equations (1.1) is a conclusion of the following system of integral equations:

$$
\left\{\begin{aligned}
x(t)= & f(t, x(t))+\frac{1}{\Gamma(\theta)} \int_{0}^{t}(t-s)^{\theta-1} h\left(s, y(s), I^{\alpha} y(s)\right) d s \\
& +\frac{1}{a_{1}-\left(b_{1}+c_{1}\right)} \frac{1}{\Gamma(\theta)} \int_{0}^{1}(1-s)^{\theta-1} \phi(s, x(s)) d s \\
& +\frac{b_{1}}{a_{1}-\left(b_{1}+c_{1}\right)} f(\eta, x(\eta))+\frac{c_{1}}{a_{1}-\left(b_{1}+c_{1}\right)} f(1, x(1)) \\
& +\frac{b_{1}}{a_{1}-\left(b_{1}+c_{1}\right)} \frac{1}{\Gamma(\theta)} \int_{0}^{\eta}(\eta-s)^{\theta-1} h\left(s, y(s), I^{\alpha} y(s)\right) d s \\
& +\frac{c_{1}}{a_{1}-\left(b_{1}+c_{1}\right)} \frac{1}{\Gamma(\theta)} \int_{0}^{1}(1-s)^{\theta-1} h\left(s, y(s), I^{\alpha} y(s)\right) d s, \\
y(t)= & f(t, y(t))+\frac{1}{\Gamma(\theta)} \int_{0}^{t}(t-s)^{\theta-1} h\left(s, x(s), I^{\alpha} x(s)\right) d s \\
& +\frac{1}{a_{2}-\left(b_{2}+c_{2}\right)} \frac{1}{\Gamma(\theta)} \int_{0}^{1}(1-s)^{\theta-1} \phi(s, y(s)) d s \\
& +\frac{b_{2}}{a_{2}-\left(b_{2}+c_{2}\right)} f(\xi, y(\xi))+\frac{c_{2}}{a_{2}-\left(b_{2}+c_{2}\right)} f(1, y(1)) \\
& +\frac{b_{2}}{a_{2}-\left(b_{2}+c_{2}\right)} \frac{1}{\Gamma(\theta)} \int_{0}^{\xi}(\xi-s)^{\theta-1} h\left(s, x(s), I^{\alpha} x(s)\right) d s \\
& +\frac{c_{2}}{a_{2}-\left(b_{2}+c_{2}\right)} \frac{1}{\Gamma(\theta)} \int_{0}^{1}(1-s)^{\theta-1} h\left(s, x(s), I^{\alpha} x(s)\right) d s .
\end{aligned}\right.
$$

Proof Applying the fractional integrable operator $I^{\theta}$ on the equation of system (1.1) and through applying Lemma 2.9 , we get

$$
x(t)=f(t, x(t))+\frac{1}{\Gamma(\theta)} \int_{0}^{t}(t-s)^{\theta-1} h\left(s, y(s), I^{\alpha} y(s)\right) d s+C_{0}+C_{1} t
$$

By applying the initial conditions $x^{\prime}(0)=0$ and $\left.\frac{\partial^{i} f(t, x(t))}{\partial t^{i}}\right|_{t=0}=0$, we obtain $C_{1}=0$ and

$$
x(t)=f(t, x(t))+\frac{1}{\Gamma(\theta)} \int_{0}^{t}(t-s)^{\theta-1} h\left(s, y(s), I^{\alpha} y(s)\right) d s+C_{0} .
$$

Now, applying the boundary conditions $a_{1} x(0)-b_{1} x(\eta)-c_{1} x(1)=\frac{1}{\Gamma(\theta)} \int_{0}^{1}(1-s)^{\theta-1} \phi(s$, $x(s)) d s$ to (3.2), we have

$$
\begin{aligned}
\left(a_{1}-b_{1}-c_{1}\right) C_{0}= & b_{1} f(\eta, x(\eta))+c_{1} f(1, x(1))+\frac{1}{\Gamma(\theta)} \int_{0}^{1}(1-s)^{\theta-1} \phi(s, x(s)) \\
& +\frac{b_{1}}{\Gamma(\theta)} \int_{0}^{\eta}(\eta-s)^{\theta-1} h\left(s, y(s), I^{\alpha} y(s)\right) d s
\end{aligned}
$$




$$
+\frac{c_{1}}{\Gamma(\theta)} \int_{0}^{1}(1-s)^{\theta-1} h\left(s, y(s), I^{\alpha} y(s)\right) d s .
$$

By rearranging, we obtain

$$
\begin{aligned}
C_{0}= & \frac{b_{1}}{a_{1}-\left(b_{1}+c_{1}\right)} f(\eta, x(\eta))+\frac{c_{1}}{a_{1}-\left(b_{1}+c_{1}\right)} f(1, x(1)) \\
& +\frac{1}{a_{1}-\left(b_{1}+c_{1}\right)} \frac{1}{\Gamma(\theta)} \int_{0}^{1}(1-s)^{\theta-1} \phi(s, x(s)) \\
& +\frac{b_{1}}{a_{1}-\left(b_{1}+c_{1}\right)} \frac{1}{\Gamma(\theta)} \int_{0}^{\eta}(\eta-s)^{\theta-1} h\left(s, y(s), I^{\alpha} y(s)\right) d s \\
& +\frac{c_{1}}{a_{1}-\left(b_{1}+c_{1}\right)} \frac{1}{\Gamma(\theta)} \int_{0}^{1}(1-s)^{\theta-1} h\left(s, y(s), I^{\alpha} y(s)\right) d s .
\end{aligned}
$$

Thus equation (3.2) becomes

$$
\begin{aligned}
x(t)= & f(t, x(t))+\frac{1}{\Gamma(\theta)} \int_{0}^{t}(t-s)^{\theta-1} h\left(s, y(s), I^{\alpha} y(s)\right) d s \\
& +\frac{1}{a_{1}-\left(b_{1}+c_{1}\right)} \frac{1}{\Gamma(\theta)} \int_{0}^{1}(1-s)^{\theta-1} \phi(s, x(s)) d s \\
& +\frac{b_{1}}{a_{1}-\left(b_{1}+c_{1}\right)} f(\eta, x(\eta))+\frac{c_{1}}{a_{1}-\left(b_{1}+c_{1}\right)} f(1, x(1)) \\
& +\frac{b_{1}}{a_{1}-\left(b_{1}+c_{1}\right)} \frac{1}{\Gamma(\theta)} \int_{0}^{\eta}(\eta-s)^{\theta-1} h\left(s, y(s), I^{\alpha} y(s)\right) d s \\
& +\frac{c_{1}}{a_{1}-\left(b_{1}+c_{1}\right)} \frac{1}{\Gamma(\theta)} \int_{0}^{1}(1-s)^{\theta-1} h\left(s, y(s), I^{\alpha} y(s)\right) d s .
\end{aligned}
$$

Analogously, following the same steps in the process for the second equation of system (1.1), we get

$$
\begin{aligned}
y(t)= & f(t, y(t))+\frac{1}{\Gamma(\theta)} \int_{0}^{t}(t-s)^{\theta-1} h\left(s, x(s), I^{\alpha} x(s)\right) d s \\
& +\frac{1}{a_{2}-\left(b_{2}+c_{2}\right)} \frac{1}{\Gamma(\theta)} \int_{0}^{1}(1-s)^{\theta-1} \psi(s, y(s)) d s \\
& +\frac{b_{2}}{a_{2}-\left(b_{2}+c_{2}\right)} f(\xi, y(\xi))+\frac{c_{2}}{a_{2}-\left(b_{2}+c_{2}\right)} f(1, y(1)) \\
& +\frac{b_{2}}{a_{2}-\left(b_{2}+c_{2}\right)} \frac{1}{\Gamma(\theta)} \int_{0}^{\xi}(\xi-s)^{\theta-1} h\left(s, x(s), I^{\alpha} x(s)\right) d s \\
& +\frac{c_{2}}{a_{2}-\left(b_{2}+c_{2}\right)} \frac{1}{\Gamma(\theta)} \int_{0}^{1}(1-s)^{\theta-1} h\left(s, x(s), I^{\alpha} x(s)\right) d s .
\end{aligned}
$$

Define the operator $F, H, T: X \times X \rightarrow X \times X$ by

$$
\begin{aligned}
& F(x, y)(t)=\left(F_{1} x(t), F_{2} y(t)\right), \\
& H(x, y)(t)=\left(H_{2} y(t), H_{1} x(t)\right), \\
& T(x, y)(t)=F(x, y)(t)+H(x, y)(t),
\end{aligned}
$$


here $F_{1}, F_{2}, H_{1}, H_{2}: X \rightarrow X$ are

$$
\begin{aligned}
F_{1} x(t)= & f(t, x(t))+\frac{b_{1}}{a_{1}-\left(b_{1}+c_{1}\right)} f(\eta, x(\eta))+\frac{c_{1}}{a_{1}-\left(b_{1}+c_{1}\right)} f(1, x(1)) \\
& +\frac{1}{a_{1}-\left(b_{1}+c_{1}\right)} \frac{1}{\Gamma(\theta)} \int_{0}^{1}(1-s)^{\theta-1} \phi(s, x(s)) d s \\
F_{2} y(t)= & f(t, y(t))+\frac{b_{2}}{a_{2}-\left(b_{2}+c_{2}\right)} f(\xi, y(\xi))+\frac{c_{2}}{a_{2}-\left(b_{2}+c_{2}\right)} f(1, y(1)) \\
& +\frac{1}{a_{2}-\left(b_{2}+c_{2}\right)} \frac{1}{\Gamma(\theta)} \int_{0}^{1}(1-s)^{\theta-1} \psi(s, y(s)) d s, \\
H_{1} x(t)= & \frac{1}{\Gamma(\theta)} \int_{0}^{t}(t-s)^{\theta-1} h\left(s, x(s), I^{\alpha} x(s)\right) d s \\
& +\frac{b_{2}}{a_{2}-\left(b_{2}+c_{2}\right)} \frac{1}{\Gamma(\theta)} \int_{0}^{\xi}(\xi-s)^{\theta-1} h\left(s, x(s), I^{\alpha} x(s)\right) d s \\
& +\frac{c_{2}}{a_{2}-\left(b_{2}+c_{2}\right)} \frac{1}{\Gamma(\theta)} \int_{0}^{1}(1-s)^{\theta-1} h\left(s, x(s), I^{\alpha} x(s)\right) d s, \\
H_{2} y(t)= & \frac{1}{\Gamma(\theta)} \int_{0}^{t}(t-s)^{\theta-1} h\left(s, y(s), I^{\alpha} y(s)\right) d s \\
& +\frac{b_{1}}{a_{1}-\left(b_{1}+c_{1}\right)} \frac{1}{\Gamma(\theta)} \int_{0}^{\eta}(\eta-s)^{\theta-1} h\left(s, y(s), I^{\alpha} y(s)\right) d s \\
& +\frac{c_{1}}{a_{1}-\left(b_{1}+c_{1}\right)} \frac{1}{\Gamma(\theta)} \int_{0}^{1}(1-s)^{\theta-1} h\left(s, y(s), I^{\alpha} y(s)\right) d s .
\end{aligned}
$$

Then the system of integral equations (3.1) can be written as an operator equation

$$
(x, y)=T(x, y)=F(x, y)+H(x, y)
$$

and fixed points of the operator equation are results of system (1.1).

Theorem 3.2 The operator $F$ is Lipschitz with constant $k$. Therefore $F$ is $\alpha$-Lipschitz with the equal constant $k$ and meets the following growth condition:

$$
\|F(x(t), y(t))\| \leq L_{F}\|(x, y)\|^{q_{2}}+M_{F} .
$$

Proof Now, we shall display that the operator $F$ is Lipschitz with constant $k$. Let $x_{1}, x_{2} \in X$, then we get

$$
\begin{aligned}
& \left|F_{1} x_{1}(t)-F_{1} x_{2}(t)\right| \\
& =\mid\left(f\left(t, x_{1}(t)\right)-f\left(t, x_{2}(t)\right)\right)+\frac{b_{1}}{a_{1}-\left(b_{1}+c_{1}\right)}\left(f\left(\eta, x_{1}(\eta)\right)-f\left(\eta, x_{2}(\eta)\right)\right) \\
& \quad+\frac{c_{1}}{a_{1}-\left(b_{1}+c_{1}\right)}\left(f\left(1, x_{1}(1)\right)-f\left(1, x_{2}(1)\right)\right) \\
& \quad+\frac{1}{a_{1}-\left(b_{1}+c_{1}\right)} \frac{1}{\Gamma(\theta)}\left(\int_{0}^{1}(1-s)^{\theta-1} \phi\left(s, x_{1}(s)\right) d s-\int_{0}^{1}(1-s)^{\theta-1} \phi\left(s, x_{2}(s)\right) d s\right) \mid \\
& \leq\left|f\left(t, x_{1}(t)\right)-f\left(t, x_{2}(t)\right)\right|
\end{aligned}
$$




$$
\begin{aligned}
& +\frac{\left|b_{1}\right|}{\left|a_{1}-\left(b_{1}+c_{1}\right)\right|}\left|f\left(\eta, x_{1}(\eta)\right)-f\left(\eta, x_{2}(\eta)\right)\right| \\
& +\frac{\left|c_{1}\right|}{\left|a_{1}-\left(b_{1}+c_{1}\right)\right|}\left|f\left(1, x_{1}(1)\right)-f\left(1, x_{2}(1)\right)\right| \\
& +\frac{1}{\left|a_{1}-\left(b_{1}+c_{1}\right)\right| \Gamma(\theta)} \int_{0}^{1}(1-s)^{\theta-1}\left|\phi\left(s, x_{1}(s)\right)-\phi\left(s, x_{2}(s)\right)\right| d s .
\end{aligned}
$$

By using conditions $\left(H_{1}\right)$ and $\left(H_{5}\right)$, we can write

$$
\begin{aligned}
\left\|F_{1} x_{1}(t)-F_{1} x_{2}(t)\right\| \leq & \lambda_{1}\left\|x_{1}-x_{2}\right\|+\frac{\left|b_{1}\right| \lambda_{1}}{\left|a_{1}-\left(b_{1}+c_{1}\right)\right|}\left\|x_{1}-x_{2}\right\| \\
& +\frac{\left|c_{1}\right| \lambda_{1}}{\left|a_{1}-\left(b_{1}+c_{1}\right)\right|}\left\|x_{1}-x_{2}\right\|+\frac{b_{\phi}}{\left|a_{1}-\left(b_{1}+c_{1}\right)\right| \Gamma(\theta+1)}\left\|x_{1}-x_{2}\right\| \\
= & {\left[\lambda_{1}+\frac{\left(\left|b_{1}\right|+\left|c_{1}\right|\right) \lambda_{1}}{\left|a_{1}-\left(b_{1}+c_{1}\right)\right|}+\frac{b_{\phi}}{\left|a_{1}-\left(b_{1}+c_{1}\right)\right| \Gamma(\theta+1)}\right]\left\|x_{1}-x_{2}\right\| } \\
= & k_{1}\left\|x_{1}-x_{2}\right\|,
\end{aligned}
$$

where $k_{1}=\lambda_{1}+\frac{\left(\left|b_{1}\right|+\left|c_{1}\right|\right) \lambda_{1}}{\left|a_{1}-\left(b_{1}+c_{1}\right)\right|}+\frac{b_{\phi}}{\left|a_{1}-\left(b_{1}+c_{1}\right)\right| \Gamma(\theta+1)}$.

Similarly,

$$
\begin{aligned}
\left\|F_{2} y_{1}(t)-F_{2} y_{2}(t)\right\| & \leq\left[\lambda_{2}+\frac{\left(\left|b_{2}\right|+\left|c_{2}\right|\right) \lambda_{2}}{\left|a_{2}-\left(b_{2}+c_{2}\right)\right|}+\frac{b_{\psi}}{\left|a_{2}-\left(b_{2}+c_{2}\right)\right| \Gamma(\theta+1)}\right]\left\|y_{1}-y_{2}\right\| \\
& =k_{2}\left\|y_{1}-y_{2}\right\|,
\end{aligned}
$$

where $k_{2}=\lambda_{2}+\frac{\left(\left|b_{2}\right|+\left|c_{2}\right|\right) \lambda_{2}}{\left|a_{2}-\left(b_{2}+c_{2}\right)\right|}+\frac{b_{\psi}}{\left|a_{2}-\left(b_{2}+c_{2}\right)\right| \Gamma(\theta+1)}$. Thus

$$
\begin{aligned}
\left\|F\left(x_{1}, y_{1}\right)-F\left(x_{2}, y_{2}\right)\right\| & =\left\|F_{1} x_{1}(t)-F_{1} x_{2}(t)\right\|+\left\|F_{2} y_{1}(t)-F_{2} y_{2}(t)\right\| \\
& \leq k_{1}\left\|x_{1}-x_{2}\right\|+k_{2}\left\|y_{1}-y_{2}\right\| \\
& \leq k\left\|\left(x_{1}, y_{1}\right)-\left(x_{2}, y_{2}\right)\right\|,
\end{aligned}
$$

where $k=\max \left(\lambda_{1}+\frac{\left(\left|b_{1}\right|+\left|c_{1}\right|\right) \lambda_{1}}{\left|a_{1}-\left(b_{1}+c_{1}\right)\right|}+\frac{b_{\phi}}{\left|a_{1}-\left(b_{1}+c_{1}\right)\right| \Gamma(\theta+1)}, \lambda_{2}+\frac{\left(\left|b_{2}\right|+\left|c_{2}\right|\right) \lambda_{2}}{\left|a_{2}-\left(b_{2}+c_{2}\right)\right|}+\frac{b_{\psi}}{\left|a_{2}-\left(b_{2}+c_{2}\right)\right| \Gamma(\theta+1)}\right)$. Then $F$ satisfies the Lipschitz condition, thus $F$ is Lipschitz with constant $k$. According to Proposition 2.4, $F$ is $\alpha$-Lipschitz with constant $k$.

Moreover, we get

$$
\begin{aligned}
\left|F_{1} x(t)\right| \leq & |f(t, x(t))|+\frac{\left|b_{1}\right|}{\left|a_{1}-\left(b_{1}+c_{1}\right)\right|}|f(\eta, x(\eta))|+\frac{\left|c_{1}\right|}{\left|a_{1}-\left(b_{1}+c_{1}\right)\right|}|f(1, x(1))| \\
& +\frac{1}{\left|a_{1}-\left(b_{1}+c_{1}\right)\right|} \frac{1}{\Gamma(\theta)} \int_{0}^{1}(1-s)^{\theta-1}|\phi(s, x(s))| d s .
\end{aligned}
$$

By $\left(H_{3}\right)$ and $\left(H_{4}\right)$, we have

$$
\begin{aligned}
\left|F_{1} x(t)\right| \leq & l_{f}\|x\|^{q_{2}}+M_{f}+\frac{\left|b_{1}\right|}{\left|a_{1}-\left(b_{1}+c_{1}\right)\right|}\left(l_{f}\|x\|^{q_{2}}+M_{f}\right) \\
& +\frac{\left|c_{1}\right|}{\left|a_{1}-\left(b_{1}+c_{1}\right)\right|}\left(l_{f}\|x\|^{q_{2}}+M_{f}\right)
\end{aligned}
$$




$$
\begin{aligned}
& +\frac{1}{\left|a_{1}-\left(b_{1}+c_{1}\right)\right|} \frac{1}{\Gamma(\theta+1)}\left(c_{\phi}\|x\|^{q_{2}}+M_{\phi}\right) \\
= & {\left[l_{f}+\frac{\left(\left|b_{1}\right|+\left|c_{1}\right|\right) l_{f}}{\left|a_{1}-\left(b_{1}+c_{1}\right)\right|}+\frac{c_{\phi}}{\left|a_{1}-\left(b_{1}+c_{1}\right)\right| \Gamma(\theta+1)}\right]\|x\|^{q_{2}} } \\
& +\left[M_{f}+\frac{\left(\left|b_{1}\right|+\left|c_{1}\right|\right) M_{f}}{\left|a_{1}-\left(b_{1}+c_{1}\right)\right|}+\frac{M_{\phi}}{\left|a_{1}-\left(b_{1}+c_{1}\right)\right| \Gamma(\theta+1)}\right] .
\end{aligned}
$$

Similarly,

$$
\begin{aligned}
\left|F_{2} y(t)\right| \leq & {\left[l_{f}+\frac{\left(\left|b_{2}\right|+\left|c_{2}\right|\right) l_{f}}{\left|a_{2}-\left(b_{2}+c_{2}\right)\right|}+\frac{c_{\psi}}{\left|a_{2}-\left(b_{2}+c_{2}\right)\right| \Gamma(\theta+1)}\right]\|y\|^{q_{2}} } \\
& +\left[M_{f}+\frac{\left(\left|b_{2}\right|+\left|c_{2}\right|\right) M_{f}}{\left|a_{2}-\left(b_{2}+c_{2}\right)\right|}+\frac{M_{\psi}}{\left|a_{2}-\left(b_{2}+c_{2}\right)\right| \Gamma(\theta+1)}\right] .
\end{aligned}
$$

Hence it follows that

$$
\begin{aligned}
\|F(x(t), y(t))\|= & \left\|\left(F_{1}(x), F_{2}(y)\right)\right\| \\
= & \left\|F_{1}(x)\right\|+\left\|F_{2}(y)\right\| \\
\leq & {\left[l_{f}+\frac{\left(\left|b_{1}\right|+\left|c_{1}\right|\right) l_{f}}{\left|a_{1}-\left(b_{1}+c_{1}\right)\right|}+\frac{c_{\phi}}{\left|a_{1}-\left(b_{1}+c_{1}\right)\right| \Gamma(\theta+1)}\right]\|x\|^{q_{2}} } \\
& +\left[l_{f}+\frac{\left(\left|b_{2}\right|+\left|c_{2}\right|\right) l_{f}}{\left|a_{2}-\left(b_{2}+c_{2}\right)\right|}+\frac{c_{\psi}}{\left|a_{2}-\left(b_{2}+c_{2}\right)\right| \Gamma(\theta+1)}\right]\|y\|^{q_{2}} \\
& +\left[M_{f}+\frac{\left(\left|b_{1}\right|+\left|c_{1}\right|\right) M_{f}}{\left|a_{1}-\left(b_{1}+c_{1}\right)\right|}+\frac{M_{\phi}}{\left|a_{1}-\left(b_{1}+c_{1}\right)\right| \Gamma(\theta+1)}\right] \\
& +\left[M_{f}+\frac{\left(\left|b_{2}\right|+\left|c_{2}\right|\right) M_{f}}{\left|a_{2}-\left(b_{2}+c_{2}\right)\right|}+\frac{M_{\psi}}{\left|a_{2}-\left(b_{2}+c_{2}\right)\right| \Gamma(\theta+1)}\right] \\
\leq & L_{F}\|(x, y)\|^{q_{2}}+M_{F},
\end{aligned}
$$

where

$$
\begin{aligned}
L_{F}= & \max \left(l_{f}+\frac{\left(\left|b_{1}\right|+\left|c_{1}\right|\right) l_{f}}{\left|a_{1}-\left(b_{1}+c_{1}\right)\right|}+\frac{c_{\phi}}{\left|a_{1}-\left(b_{1}+c_{1}\right)\right| \Gamma(\theta+1)},\right. \\
& \left.l_{f}+\frac{\left(\left|b_{2}\right|+\left|c_{2}\right|\right) l_{f}}{\left|a_{2}-\left(b_{2}+c_{2}\right)\right|}+\frac{c_{\psi}}{\left|a_{2}-\left(b_{2}+c_{2}\right)\right| \Gamma(\theta+1)}\right), \\
M_{F}= & 2 \max \left(M_{f}+\frac{\left(\left|b_{1}\right|+\left|c_{1}\right|\right) M_{f}}{\left|a_{1}-\left(b_{1}+c_{1}\right)\right|}+\frac{M_{\phi}}{\left|a_{1}-\left(b_{1}+c_{1}\right)\right| \Gamma(\theta+1)},\right. \\
& \left.M_{f}+\frac{\left(\left|b_{2}\right|+\left|c_{2}\right|\right) M_{f}}{\left|a_{2}-\left(b_{2}+c_{2}\right)\right|}+\frac{M_{\psi}}{\left|a_{2}-\left(b_{2}+c_{2}\right)\right| \Gamma(\theta+1)}\right) .
\end{aligned}
$$

Theorem 3.3 The operator $H: X \times X \rightarrow X \times X$ is continuous and meets the following growth condition:

$$
\|H(x(t), y(t))\| \leq L_{H}\|(x, y)\|^{q_{1}}+M_{H} .
$$


Proof Consider a bounded subset of $X \times X$ as

$$
B_{r}=\{\|(x, y)\| \leq r:(x, y) \in X \times X\} \subseteq X \times X .
$$

Let $\left\{\left(x_{n}, y_{n}\right)\right\}$ be a sequence in $B_{r}$ such that $\left(x_{n}, y_{n}\right) \rightarrow(x, y)$ as $n \rightarrow \infty$. To show that $H$ is continuous, we consider

$$
\begin{aligned}
& \left|H_{1}\left(x_{n}\right)-H_{1}(x)\right| \\
& \leq \leq \frac{1}{\Gamma(\theta)} \int_{0}^{t}(t-s)^{\theta-1}\left|h\left(s, x_{n}(s), I^{\alpha} x_{n}(s)\right)-h\left(s, x(s), I^{\alpha} x(s)\right)\right| d s \\
& \quad+\frac{\left|b_{2}\right|}{\left|a_{2}-\left(b_{2}+c_{2}\right)\right|} \frac{1}{\Gamma(\theta)} \int_{0}^{\xi}(\xi-s)^{\theta-1}\left|h\left(s, x_{n}(s), I^{\alpha} x_{n}(s)\right)-h\left(s, x(s), I^{\alpha} x(s)\right)\right| d s \\
& \quad+\frac{\left|c_{2}\right|}{\left|a_{2}-\left(b_{2}+c_{2}\right)\right|} \frac{1}{\Gamma(\theta)} \int_{0}^{1}(1-s)^{\theta-1}\left|h\left(s, x_{n}(s), I^{\alpha} x_{n}(s)\right)-h\left(s, x(s), I^{\alpha} x(s)\right)\right| d s .
\end{aligned}
$$

From the continuity of $h$, it follows that

$$
h\left(s, x_{n}(s), I^{\alpha} x_{n}(s)\right) \rightarrow h\left(s, x(s), I^{\alpha} x(s)\right) \quad \text { as } n \rightarrow \infty .
$$

For every $t \in[0,1]$, by applying $\left(H_{2}\right)$, and the Lebesgue dominated convergent theorem, we can get

$$
\int_{0}^{t} \frac{(t-s)^{\theta-1}}{\Gamma(\theta)}\left|h\left(s, x_{n}(s), I^{\alpha} x_{n}(s)\right)-h\left(s, x(s), I^{\alpha} x(s)\right)\right| d s \rightarrow 0 \quad \text { as } n \rightarrow \infty
$$

The same as the other terms approach 0 as $n \rightarrow \infty$, thus

$$
\left\|H_{1}\left(x_{n}\right)-H_{1}(x)\right\| \rightarrow 0 \quad \text { as } n \rightarrow \infty .
$$

Then $H_{1}$ is continuous. By the same steps as above, one lightly gets that

$$
\left\|H_{2}\left(y_{n}\right)-H_{2}(y)\right\| \rightarrow 0 \quad \text { as } n \rightarrow \infty
$$

That is, $\mathrm{H}_{2}$ is continuous. From (3.3) and (3.4), we have

$$
\left\|H\left(x_{n}, y_{n}\right)-H(x, y)\right\| \rightarrow 0 \quad \text { as } n \rightarrow \infty,
$$

which means that $H$ is continuous.

Moreover, by $\left(\mathrm{H}_{2}\right)$, we have

$$
\begin{aligned}
\left|H_{1} x(t)\right|= & \mid \frac{1}{\Gamma(\theta)} \int_{0}^{t}(t-s)^{\theta-1} h\left(s, x(s), I^{\alpha} x(s)\right) d s \\
& +\frac{b_{2}}{a_{2}-\left(b_{2}+c_{2}\right)} \frac{1}{\Gamma(\theta)} \int_{0}^{\xi}(\xi-s)^{\theta-1} h\left(s, x(s), I^{\alpha} x(s)\right) d s \\
& +\frac{c_{2}}{a_{2}-\left(b_{2}+c_{2}\right)} \frac{1}{\Gamma(\theta)} \int_{0}^{1}(1-s)^{\theta-1} h\left(s, x(s), I^{\alpha} x(s)\right) d s \mid
\end{aligned}
$$




$$
\begin{aligned}
\leq & \frac{t^{\theta}}{\Gamma(\theta+1)}\left(l_{h}^{1}\|x\|^{q_{1}}+l_{h}^{2}\left\|I^{\alpha} x\right\|^{q_{1}}+M_{h}\right) \\
& +\frac{\left|b_{2}\right| \xi^{\theta}}{\left|a_{2}-\left(b_{2}+c_{2}\right)\right| \Gamma(\theta+1)}\left(l_{h}^{1}\|x\|^{q_{1}}+l_{h}^{2}\left\|I^{\alpha} x\right\|^{q_{1}}+M_{h}\right) \\
& +\frac{\left|c_{2}\right|}{\left|a_{2}-\left(b_{2}+c_{2}\right)\right| \Gamma(\theta+1)}\left(l_{h}^{1}\|x\|^{q_{1}}+l_{h}^{2}\left\|I^{\alpha} x\right\|^{q_{1}}+M_{h}\right) \\
\leq & {\left[\frac{1}{\Gamma(\theta+1)}+\frac{\left|b_{2}\right|+\left|c_{2}\right|}{\left|a_{2}-\left(b_{2}+c_{2}\right)\right| \Gamma(\theta+1)}\right]\left(l_{h}^{1}\|x\|^{q_{1}}+l_{h}^{2}\left\|I^{\alpha} x\right\|^{q_{1}}+M_{h}\right) . }
\end{aligned}
$$

Similarly,

$$
\left|H_{2} y(t)\right| \leq\left[\frac{1}{\Gamma(\theta+1)}+\frac{\left|b_{1}\right|+\left|c_{1}\right|}{\left|a_{1}-\left(b_{1}+c_{1}\right)\right| \Gamma(\theta+1)}\right]\left(l_{h}^{1}\|y\|^{q_{1}}+l_{h}^{2}\left\|I^{\alpha} y\right\|^{q_{1}}+M_{h}\right) .
$$

Thus

$$
\begin{aligned}
\|H(x(t), y(t))\|= & \left\|H_{1} x(t)\right\|+\left\|H_{2} y(t)\right\| \\
\leq & {\left[\frac{1}{\Gamma(\theta+1)}+\frac{\left|b_{2}\right|+\left|c_{2}\right|}{\left|a_{2}-\left(b_{2}+c_{2}\right)\right| \Gamma(\theta+1)}\right]\left(l_{h}^{1}\|x\|^{q_{1}}+l_{h}^{2}\left\|I^{\alpha} x\right\|^{q_{1}}+M_{h}\right) } \\
& +\left[\frac{1}{\Gamma(\theta+1)}+\frac{\left|b_{1}\right|+\left|c_{1}\right|}{\left|a_{1}-\left(b_{1}+c_{1}\right)\right| \Gamma(\theta+1)}\right]\left(l_{h}^{1}\|y\|^{q_{1}}+l_{h}^{2}\left\|I^{\alpha} y\right\|^{q_{1}}+M_{h}\right) \\
\leq & {\left[\frac{1}{\Gamma(\theta+1)}+\frac{\left|b_{2}\right|+\left|c_{2}\right|}{\left|a_{2}-\left(b_{2}+c_{2}\right)\right| \Gamma(\theta+1)}\right]\left[l\left(\|x\|^{q_{1}}+\left\|I^{\alpha} x\right\|^{q_{1}}\right)+M_{h}\right] } \\
& +\left[\frac{1}{\Gamma(\theta+1)}+\frac{\left|b_{1}\right|+\left|c_{1}\right|}{\left|a_{1}-\left(b_{1}+c_{1}\right)\right| \Gamma(\theta+1)}\right]\left[l\left(\|y\|^{q_{1}}+\left\|I^{\alpha} y\right\|^{q_{1}}\right)+M_{h}\right] \\
\leq & L_{h}\left[\|x\|^{q_{1}}+\|y\|^{q_{1}}+\left\|I^{\alpha} x\right\|^{q_{1}}+\left\|I^{\alpha} y\right\|^{q_{1}}+2 M_{h}\right] \\
\leq & L_{H}\|(x, y)\|^{q_{1}}+M_{H},
\end{aligned}
$$

where $l=\max \left\{l_{h}^{1}, l_{h}^{2}\right\} \in[0,1), L_{h}=\max \left(\frac{1}{\Gamma(\theta+1)}+\frac{\left|b_{2}\right|+\left|c_{2}\right|}{\left|a_{2}-\left(b_{2}+c_{2}\right)\right| \Gamma(\theta+1)}, \frac{1}{\Gamma(\theta+1)}+\frac{\left|b_{1}\right|+\left|c_{1}\right|}{\left|a_{1}-\left(b_{1}+c_{1}\right)\right| \Gamma(\theta+1)}\right)$, $L_{H}=\left(1+\left(\frac{1}{\Gamma(\alpha+1)}\right)^{q_{1}}\right) L_{h}, M_{H}=2 L_{h} M_{h}$. Hence $H$ satisfies the growth condition.

Theorem 3.4 The operator $H: X \times X \rightarrow X \times X$ is compact.

Proof Let $\Omega$ be a bounded subset of $B_{r} \subseteq X \times X$ and $\left\{\left(x_{n}, y_{n}\right)\right\}$ be a sequence in $\Omega$, through applying the growth condition of $H$, it is obvious that $H(\Omega)$ is uniformly bounded in $X \times X$. Now, we need to reveal that $H$ is equicontinuous. Let $0 \leq t \leq \tau \leq 1$, then we obtain

$$
\begin{aligned}
\left|H_{1} x_{n}(t)-H_{1} x_{n}(\tau)\right|= & \mid \frac{1}{\Gamma(\theta)} \int_{0}^{t}(t-s)^{\theta-1} h\left(s, x_{n}(s), I^{\alpha} x_{n}(s)\right) d s \\
& -\frac{1}{\Gamma(\theta)} \int_{0}^{\tau}(\tau-s)^{\theta-1} h\left(s, x_{n}(s), I^{\alpha} x_{n}(s)\right) d s \mid \\
= & \mid \frac{1}{\Gamma(\theta)} \int_{0}^{t}\left[(t-s)^{\theta-1}-(\tau-s)^{\theta-1}\right] h\left(s, x_{n}(s), I^{\alpha} x_{n}(s)\right) d s \\
& -\frac{1}{\Gamma(\theta)} \int_{t}^{\tau}(\tau-s)^{\theta-1} h\left(s, x_{n}(s), I^{\alpha} x_{n}(s)\right) d s \mid
\end{aligned}
$$




$$
\begin{aligned}
\leq & \frac{1}{\Gamma(\theta)} \int_{0}^{t}\left[(t-s)^{\theta-1}-(\tau-s)^{\theta-1}\right]\left|h\left(s, x_{n}(s), I^{\alpha} x_{n}(s)\right)\right| d s \\
& +\frac{1}{\Gamma(\theta)} \int_{t}^{\tau}(\tau-s)^{\theta-1}\left|h\left(s, x_{n}(s), I^{\alpha} x_{n}(s)\right)\right| d s \\
\leq & \frac{l_{h}^{1}\left\|x_{n}\right\|^{q_{1}}+l_{h}^{2}\left\|I^{\alpha} x_{n}\right\|^{q_{1}}+M_{h}}{\Gamma(\theta+1)}\left[t^{\theta}-\tau^{\theta}+2(\tau-t)^{\theta}\right] .
\end{aligned}
$$

Similarly,

$$
\left|H_{2} y_{n}(t)-H_{2} y_{n}(\tau)\right| \leq \frac{l_{h}^{1}\left\|y_{n}\right\|^{q_{1}}+l_{h}^{2}\left\|I^{\alpha} y_{n}\right\|^{q_{1}}+M_{h}}{\Gamma(\theta+1)}\left[t^{\theta}-\tau^{\theta}+2(\tau-t)^{\theta}\right] .
$$

Taking limit as $t \rightarrow \tau$, we get

$$
\left\|H_{1} x_{n}(t)-H_{1} x_{n}(\tau)\right\| \rightarrow 0
$$

and

$$
\left\|H_{2} y_{n}(t)-H_{2} y_{n}(\tau)\right\| \rightarrow 0
$$

which implies that

$$
\left\|H\left(x_{n}, y_{n}\right)(t)-H\left(x_{n}, y_{n}\right)(\tau)\right\| \rightarrow 0 .
$$

This reveals that $H(x, y)$ is equicontinuous. $H(x, y)$ is compact by the Arzelá-Ascoli theorem. Hence, according to Proposition 2.5, $H$ is $\alpha$-Lipschitz with constant zero.

Theorem 3.5 If $\left(H_{1}\right)-\left(H_{5}\right)$ hold and

$$
\begin{aligned}
k= & \max \left(\lambda_{1}+\frac{\left(\left|b_{1}\right|+\left|c_{1}\right|\right) \lambda_{1}}{\left|a_{1}-\left(b_{1}+c_{1}\right)\right|}+\frac{b_{\phi}}{\left|a_{1}-\left(b_{1}+c_{1}\right)\right| \Gamma(\theta+1)},\right. \\
& \left.\lambda_{2}+\frac{\left(\left|b_{2}\right|+\left|c_{2}\right|\right) \lambda_{2}}{\left|a_{2}-\left(b_{2}+c_{2}\right)\right|}+\frac{b_{\psi}}{\left|a_{2}-\left(b_{2}+c_{2}\right)\right| \Gamma(\theta+1)}\right) \in[0,1),
\end{aligned}
$$

then coupled system (1.1) has at least one solution $(x, y) \in X \times X$. And the solution set of (1.1) is bounded in $X \times X$.

Proof From Theorem 3.2 and $k \in[0,1), F$ is $\alpha$-Lipschitz with constant $k \in[0,1)$, according to Theorem 3.4, $H$ is $\alpha$-Lipschitz with constant 0. By Proposition 2.3 and Definition 2.2, $T$ is a strict $\alpha$-contraction with constant $k$. Hence, $T$ is $\alpha$-condensing. Then, we think over the following set:

$$
R=\{(x, y) \in X \times X: \text { there exist } \zeta \in[0,1],(x, y)=\zeta T(x, y)\} .
$$

We have to reveal that $R$ is bounded in $X \times X$. Let $(x, y) \in R$, then by applying the growth conditions of Theorem 3.2 and Theorem 3.3, we have

$$
(x, y)=\zeta T(x, y)=\zeta(F(x, y)+H(x, y))
$$


thus

$$
\begin{aligned}
\|(x, y)\| & =\zeta\|T(x, y)\| \\
& \leq \zeta(\|F(x, y)\|+\|H(x, y)\|) \\
& \leq \zeta\left[L_{F}\|(x, y)\|^{q_{2}}+M_{F}+L_{H}\|(x, y)\|^{q_{1}}+M_{H}\right] \\
& =\zeta\left(L_{F}\|(x, y)\|^{q_{2}}+L_{H}\|(x, y)\|^{q_{1}}\right)+\zeta\left(M_{F}+M_{H}\right),
\end{aligned}
$$

where $q_{1}, q_{2} \in[0,1)$. Thus $R$ is bounded in $X \times X$. According to Theorem 2.6, there exists $r>0$ such that $R \subset B_{r}(0)$, then

$$
\left.D\left(I-\zeta T, B_{r}(0), 0\right)\right)=1, \quad \text { for all } \zeta \in[0,1]
$$

Therefore, $T$ has at least one fixed point, then coupled system (1.1) has at least one solution.

\section{Examples}

This part, we have the following example account for our main results.

Example 4.1 Give thought to the following equation:

$$
\begin{cases}D^{\frac{3}{2}}\left[x(t)-\frac{\sin ^{2}(t)|x(t)|}{5(2(\mid)|x(t)|}\right]=\frac{e^{-\pi t}}{10}+\frac{\cos |y(t)|+\sin |y(t)|}{51+t^{2}}, & t \in[0,1], \\ D^{\frac{3}{2}}\left[y(t)-\frac{\sin ^{2}(t)|y(t)|}{5(2+|y(t)|)}\right]=\frac{e^{-\pi t}}{10}+\frac{\cos |x(t)|+\sin |x(t)|}{51+t^{2}}, & t \in[0,1], \\ x^{\prime}(0)=y^{\prime}(0)=0, & \\ \frac{1}{4} x(0)-\frac{1}{2} x\left(\frac{1}{2}\right)-6 x(1)=\frac{1}{\Gamma\left(\frac{3}{2}\right)} \int_{0}^{1}(1-s)^{\frac{1}{2}} \frac{\sin x(s)}{2} d s, \\ \frac{1}{5} y(0)-\frac{1}{7} y\left(\frac{1}{2}\right)-8 y(1)=\frac{1}{\Gamma\left(\frac{3}{2}\right)} \int_{0}^{1}(1-s)^{\frac{1}{2}} \frac{\cos y(s)}{5} d s,\end{cases}
$$

where $h=\frac{e^{-\pi t}}{10}+\frac{\cos |y(t)|+\sin |y(t)|}{51+t^{2}}, \theta=\frac{3}{2}, a_{1}=\frac{1}{4}, b_{1}=\frac{1}{2}, c_{1}=6, a_{2}=\frac{1}{5}, b_{2}=\frac{1}{7}, c_{2}=8, \eta=\xi=\frac{1}{2}$. Let $\zeta=\frac{1}{5}$, then by routine calculation, we can have $c_{\phi}=b_{\phi}=\frac{1}{2}, c_{\psi}=b_{\psi}=\frac{1}{5}, M_{\phi}=M_{\psi}=0$, $l_{h}^{1}=l_{h}^{2}=\frac{1}{51}, M_{h}=\frac{1}{10}, l_{f}=\frac{1}{10}, M_{f}=0, \lambda_{1}=\lambda_{2}=\frac{1}{10}$. Thus assumptions $\left(H_{1}\right)-\left(H_{5}\right)$ hold, and

$$
\begin{aligned}
& \lambda_{1}+\frac{\left(\left|b_{1}\right|+\left|c_{1}\right|\right) \lambda_{1}}{\left|a_{1}-\left(b_{1}+c_{1}\right)\right|}+\frac{b_{\phi}}{\left|a_{1}-\left(b_{1}+c_{1}\right)\right| \Gamma(\theta+1)} \approx 0.389, \\
& \left.\lambda_{2}+\frac{\left(\left|b_{2}\right|+\left|c_{2}\right|\right) \lambda_{2}}{\left|a_{2}-\left(b_{2}+c_{2}\right)\right|}+\frac{b_{\psi}}{\left|a_{2}-\left(b_{2}+c_{2}\right)\right| \Gamma(\theta+1)}\right) \approx 0.221 .
\end{aligned}
$$

Next,

$$
\begin{aligned}
\left|F\left(x_{1}, y_{1}\right)(t)-F\left(x_{2}, y_{2}\right)(t)\right| \leq & \frac{1}{10}\left|x_{1}(t)-x_{2}(t)\right|+\frac{1}{10}\left|y_{1}(t)-y_{2}(t)\right| \\
& +\frac{1}{11.078} \int_{0}^{1}(1-s)^{\frac{1}{2}}\left|\sin \left(x_{1}\right)-\sin \left(x_{2}\right)\right| d s \\
& +\frac{1}{35.196} \int_{0}^{1}(1-s)^{\frac{1}{2}}\left|\cos \left(y_{1}\right)-\cos \left(y_{2}\right)\right| d s \\
\leq & 0.160\left\|x_{1}-x_{2}\right\|+0.119\left\|y_{1}-y_{2}\right\|
\end{aligned}
$$




$$
\leq 0.160\left\|\left(x_{1}, y_{1}\right)-\left(x_{2}, y_{2}\right)\right\|,
$$

thus $F$ is $\alpha$-Lipschitz with the constant 0.160 , and thus $H$ is $\alpha$-Lipschitz with the constant zero, which means that $T$ is $\alpha$-Lipschitz with the constant 0.160 . Since

$$
R=\{(x, y) \in X \times X: \text { there exist } \zeta \in[0,1],(x, y)=\zeta T(x, y)\}
$$

then, by routine calculation, we obtain $L_{F}=0.216, L_{H}=2.380, M_{F}=0, M_{H}=0.238$.

Thus

$$
\|(x, y)\| \cong 0.703 \leq 1
$$

then $R$ is bounded, through Theorem 3.5, then problem (4.1) has at least one solution.

\section{Acknowledgements}

The authors are very grateful to the referees for their very helpful comments and suggestions, which greatly improved the presentation of this paper.

\section{Funding}

This research is funded by the National Natural Science Foundation of P.R. China (No:11661037).

\section{Availability of data and materials}

No data were used to support this study.

\section{Competing interests}

The authors declare that they have no competing interests.

\section{Authors' contributions}

Both authors made equal contribution. All authors read and approved the final manuscript.

\section{Publisher's Note}

Springer Nature remains neutral with regard to jurisdictional claims in published maps and institutional affiliations.

Received: 21 March 2021 Accepted: 10 June 2021 Published online: 26 June 2021

\section{References}

1. Kilbas, A.A., Srivastava, H.M., Trujillo, J.J.: Theory and Applications of Fractional Differential Equations. Elsevier, The Netherlands (2006)

2. Hilfer, R.: Applications of Fractional Calculus in Physics. World Scientific, Singapore (2000)

3. Freed, A.D., Diethelm, K.: Fractional calculus in biomechanics: a 3D viscoelastic model using regularized fractional-derivative kernels with application to the human calcaneal fat pad. Biomech. Model. Mechanobiol. 5 203-215 (2006)

4. Magin, R.L.: Fractional calculus in bioengineering, part 1. Crit. Rev. Biomed. Eng. 32, 1-104 (2004)

5. Ge, Z.M., Ou, C.Y.: Chaos synchronization of fractional order modified Duffing systems with parameters excited by a chaotic signal. Chaos Solitons Fractals 35, 705-717 (2008)

6. Sokolov, I.M., Klafter, J., Blumen, A.: Fractional kinetics. Phys. Today 55, 48-55 (2002)

7. Petrá, I., Magin, R.L.: Simulation of drug uptake in a two compartmental fractional model for a biological system. Commun. Nonlinear Sci. Numer. Simul. 16, 4588-4595 (2011)

8. Xie, D.P., Bai, C.Z., Liu, Y.: Positive solutions for a coupled system of semipositone fractional differential equations with the integral boundary conditions. Eur. Phys. J. Spec. Top. 226, 3551-3566 (2017)

9. Zhang, X.Q., Wang, L., Sun, Q.: Existence of positive solutions for a class of nonlinear fractional differential equations with integral boundary conditions and a parameter. J. Appl. Math. Comput. 226, 708-718 (2014)

10. Sun, Q., Ji, H.W., Cui, Y.J.: Positive solutions for boundary value problems of fractional differential equation with integral boundary conditions. J. Funct. Spaces 2018, 6461930 (2018)

11. Wang, Y., Liu, L.S., Wu, Y.H.: Positive solutions for a class of higher-order singular semipositone fractional differential systems with coupled integral boundary conditions and parameters. Adv. Differ. Equ. 2014, 268 (2014)

12. Yang, W.G.: Positive solutions for singular coupled integral boundary value problems of nonlinear Hadamard fractional differential equations. J. Nonlinear Sci. Appl. 8, 110-129(2015)

13. Jiang, J.Q., Liu, W.W., Wang, H.C.: Positive solutions for higher order nonlocal fractional differential equation with integral boundary conditions. J. Funct. Spaces 2018, 1-12 (2018)

14. Ali, I., Haq, S., Nisar, K.S., Baleanu, D.: An efficient numerical scheme based on Lucas polynomials for the study of multidimensional Burgers-type equations. Adv. Differ. Equ. 2021, 43 (2021) 
15. Xie, J.L., Duan, L.J.: Existence of solutions for fractional differential equations with $p$-Laplacian operator and integral boundary conditions. J. Funct. Spaces 100, 1-7 (2020)

16. Seemab, A., Rehman, M.U., Alzabut, J., Hamdi, A.: On the existence of positive solutions for generalized fractional boundary value problems. Bound. Value Probl. 2019, 186 (2019)

17. Zhao, K.H., Gong, P.: Positive solutions for impulsive fractional differential equations with generalized periodic boundary value conditions. Adv. Differ. Equ. 2014, 255 (2014)

18. Yang, C., Zhai, C.B., Zhang, L.L.: Local uniqueness of positive solutions for a coupled system of fractional differential equations with integral boundary conditions. Adv. Differ. Equ. 2017, 282 (2017)

19. Zhang, W., Liu, W.B.: Existence of solutions for several higher-order Hadamard-type fractional differential equations with integral boundary conditions on infinite interval. Bound. Value Probl. 2018, 134 (2018)

20. Liu, Z.H., Ding, Y.Z., Liu, C.W., Zhao, C.Y.: Existence and uniqueness of solutions for singular fractional differential equation boundary value problem with $p$-Laplacian. Bound. Value Probl. 2020, 83 (2020)

21. Zhang, W., Ni, J.B.: New multiple positive solutions for Hadamard-type fractional differential equations with nonlocal conditions on an infinite interval. Appl. Math. Lett. 118, 107165 (2021)

22. Sher, M., Shah, K., Feckan, M., Khan, R.A.: Qualitative analysis of multi-terms fractional order delay differential equations via the topological degree theory. Mathematics 8, 218 (2020)

23. Bashiri, T., Vaezpour, S.M., Park, C.: A coupled fixed point theorem and application to fractional hybrid differential problems. Fixed Point Theory Appl. 2016, 23 (2016)

24. Ahmad, I., Shah, K., Rahman, G.U., Baleanu, D.: Stability analysis for a nonlinear coupled system of fractional hybrid delay differential equations. Math. Methods Appl. Sci. 43(15), 8669-8682 (2020)

25. Muthaiah, S., Baleanu, D., Thangaraj, N.G.: Existence and Hyers-Ulam type stability results for nonlinear coupled system of Caputo-Hadamard type fractional differential equations. AIMS Math. 6(1), 168-194 (2021)

26. Deimling, K.: Nonlinear Functional Analysis. World Publishing Corporation, (1980)

27. Isaia, F.: On a nonlinear integral equation without compactness. Acta Math. Univ. Comen. 75, 233-240 (2006)

28. Shah, K., Khan, P.A.: Existence and uniqueness of positive solutions to a coupled system of nonlinear fractional order differential equations with anti periodic boundary conditions. Differ. Equ. Appl. 7, 245-262 (2015)

\section{Submit your manuscript to a SpringerOpen ${ }^{\circ}$ journal and benefit from:}

- Convenient online submission

- Rigorous peer review

- Open access: articles freely available online

- High visibility within the field

- Retaining the copyright to your article

Submit your next manuscript at $>$ springeropen.com 